Suturing the conduit directly to the apex without using an apical connector seemed very effective, especially in terms of the control of bleeding from the friable apex. It enabled us to take deep bites through the epicardium and the endocardium, allowing excellent integrity between the left ventricle and the graft. In the present case bleeding from the proximal anastomosis was not a problem at all.

This technique has additional merits. Crossclamping of the aorta is never required. It does not compromise major coronary arteries, other valves, or the conduction system. ${ }^{1,3}$ Paravalvular leak and patient-prosthesis mismatch are completely avoided.

Apicoaortic conduit for patients after $\mathrm{CABG}$ with acquired aortic stenosis is feasible but requires special attention, especially when coring the ventricle. With more modifications, this method has the possibility for a more common use.

\section{References}

1. Cooley DA, Lopez RM, Absi TS. Apicoaortic conduit for left ventricular outflow tract obstruction: revisited. Ann Thorac Surg. 2000;69: $1511-4$.

2. Lockowandt U. Apicoaortic valved conduit: potential for progress? J Thorac Cardiovasc Surg. 2006;132:796-801.

3. Gammie JS, Brown JW, Brown JM, Poston RS, Pierson RN III Odonkor PN, et al. Aortic valve bypass for the high-risk patient with aortic stenosis. Ann Thorac Surg. 2006;81:1605-11.

4. White CS, Jeudy J, Read K, Brown JM, Griffith BP, Gammie JS. Aortic valve bypass for aortic stenosis: imaging appearances on multidetector CT. Int J Cardiovasc Imaging. 2006. Epub ahead of print.

5. Yamaura Y, Yoshikawa J, Yoshida K, Akasaka T. Echocardiographic findings of subepicardial aneurysm of the left ventricle. Am Heart $J$. $1994 ; 127: 211-4$

\title{
Arterial coronary-coronary conduit over single, distal left anterior descending coronary artery lesion: 3.5 years afterward
}

Duško G. Nežić, MD, PhD, FETCS, Aleksandar M. Knežević, MD, BSc, Milan V. Ćirković, MD, Saša D. Borović, MD, and

Predrag S. Milojević, MD, PhD, FETCS, Belgrade, Serbia

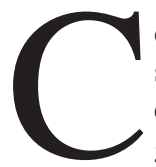

oronary-coronary bypass grafting $(\mathrm{CCBG})$ is occasionally performed in cases of heavily calcified ascending aorta and inadequate length of available grafts, ${ }^{1}$ and, sometimes, to bypass lesions on large coronary arteries. ${ }^{2}$ Although early patency rates are reported to be excellent, ${ }^{1}$ very few data are available on the midterm or long-term angiographic patency rates of these grafts. We present the midterm follow-up of a coronary-coronary arterial bypass performed 3.5 years earlier over a single, distal stenosis on the patient's left anterior descending (LAD) coronary artery.

\section{Clinical Summary}

In July 2003, a 41-year-old man was admitted at our hospital with progressive angina. Coronary angiography performed on that occasion had demonstrated an occluded right coronary artery, a $75 \%$ stenosis of the ramus intermedius, and long $(3 \mathrm{~cm}$

\footnotetext{
From the Department of Cardiac Surgery, "Dedinje" Cardiovascular Institute, Belgrade, Serbia.

Received for publication March 7, 2007; accepted for publication March 15, 2007.

Address for reprints: Duško Nežić, MD, PhD, FETCS, Chief, Department of Cardiac Surgery I, Dedinje Cardiovascular Institute, M. Tepića 1, 11040 Belgrade, Serbia (E-mail: nezic@EUnet.yu).

J Thorac Cardiovasc Surg 2007;134:238-40

$0022-5223 / \$ 32.00$

Copyright (C) 2007 by The American Association for Thoracic Surgery doi:10.1016/j.jtcvs.2007.03.009
}

in length) - up to $95 \%$ stenosis on the border zone between the mid and distal thirds of the large LAD. By-

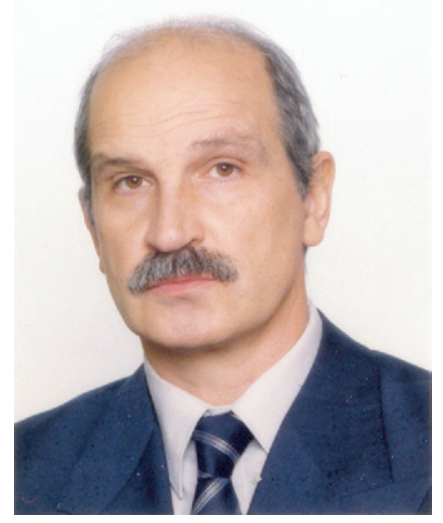

Dr Nežić pass surgery with a pedicled left internal thoracic artery (LITA) and vein graft was planned and accomplished. The vein graft was used to bypass the right coronary system. Because there was no proximal stenosis on the LAD, we decided to use only a short, free segment of the pedicled LITA to perform a coronary-coronary bypass (proximal and distal connections were done as a termino-lateral anastomosis) over that single, distal stenosis. We also grafted the in situ LITA remnant onto the ramus intermedius. The patient's postoperative course and convalescence progressed without any difficulty. A predischarge angiogram showed a patent, free LITA coronary-coronary conduit $(\mathrm{CCC})^{2}$ (Figure 1).

The patient has been recently readmitted for evaluation of chest discomfort. Although results of the stress test were negative, he voluntarily underwent coronary angiography. According to visual assessment by the Core Angiography Committee (two cardiologists and a cardiac surgeon, to whom the angiogram was presented in masked fashion concerning the existence of the CCC on the LAD), vein and LITA conduits were declared to be perfectly patent. The LAD coronary artery was described as having no lesions. Indeed, the caliber of the LITA free segment incorporated into the LAD coronary bed in coronary-coronary fashion can be seen to match (Figure 2) that of native coronary artery very well, so much so that it is difficult 


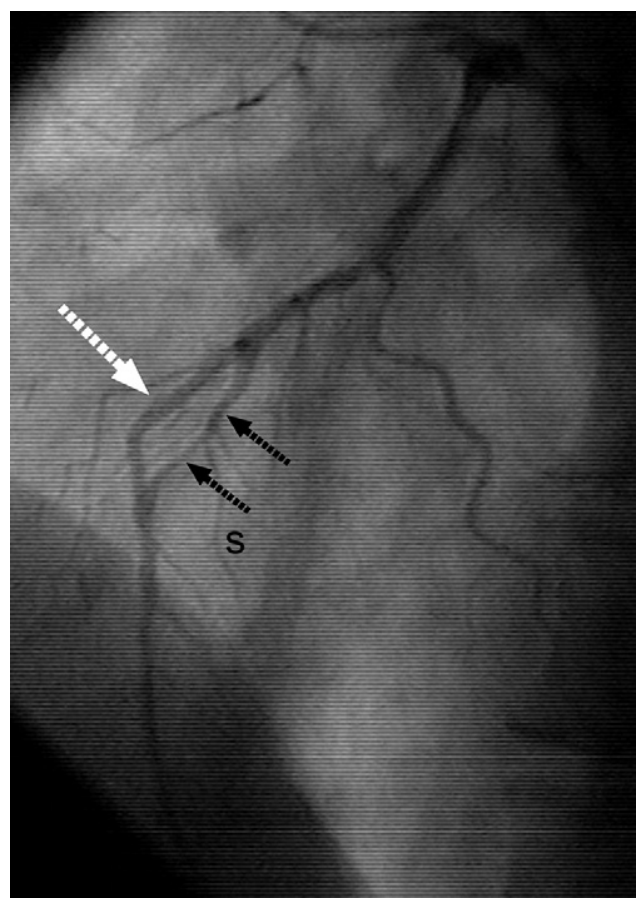

Figure 1. Postoperative angiogram of our patient (left anterior oblique view) showing patent, free LITA CCC (white dotted arrow), and bypassed native LAD region with severe stenosis (black dotted arrows) and septal branch (S).

to observe the presence of a conduit in the coronary circulation. To the best of our knowledge, this is a unique angiogram confirming midterm patency (3.5 years) of arterial CCC over a single LAD lesion.

\section{Discussion}

Primarily used in the beginning of aorta-coronary bypass surgery (termino-terminal interposition of saphenous vein grafts between two parts of resected coronary artery), CCBG was revised by Biglioli and associates ${ }^{3}$ later on, and from the hemodynamic point of view, the physiologic restoration of coronary blood flow has been confirmed. Excellent results of the largest series on $\mathrm{CCBG}^{1}$ have confirmed the capability of this technique to play an important role in coronary surgery.

Very few data are available on the midterm or long-term outcome of CCCs. Mariscalco and colleagues ${ }^{4}$ have recently reported 19-year patency of a coronary-coronary venous bypass graft. The only report covering late patency of an arterial coronary-coronary graft is by Barboso and Rusticali. ${ }^{5}$ They used a free LITA segment to bypass distal LAD stenosis in 6 patients (LAD presented with two stenoses, proximal and distal in all patients), while the in situ LITA remnants grafted a proximal stenosis on the same LADs. Late angiograms were performed in only 2 patients ( 3 and 8 years postoperatively), recording patent arterial CCCs. However, the inflow of those CCCs was obtained from the midpoint of the LAD, subserved itself by the in situ LITA

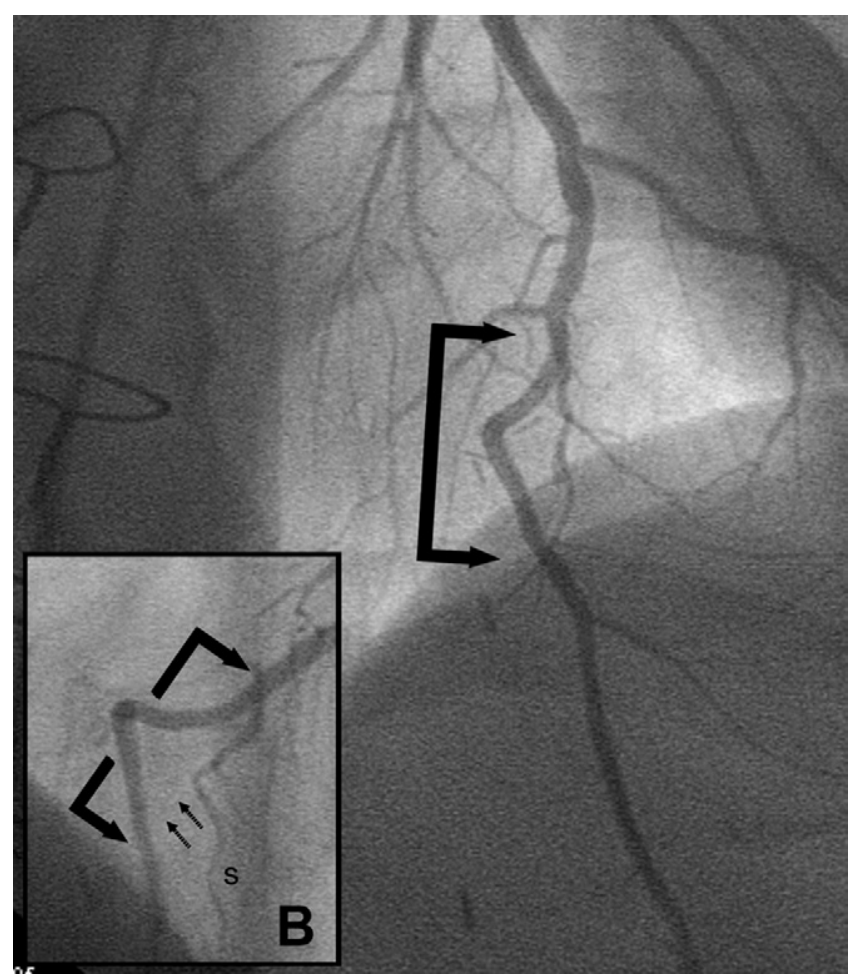

Figure 2. Angiogram in the same patient 3.5 years afterward showing patent arterial CCC (double black arrow) incorporated into the LAD coronary bed. B, Patent, free LITA CCC (double black arrow). Occluded distal part of previously bypassed native LAD region (black arrows) with still patent septal branch (S).

remnant. Thus, those CCCs were governed by an aorta-coronary physiology. The physiologic benefit (ie, diastolic blood flow closer to physiologic norms) of CCBG can be achieved only when the origin and initial segment of the targeted artery are free of atherosclerotic lesions. In such a case we can use this region as a site of proximal anastomosis in a CCBG procedure, thus using the advantages of the coronary prompting effect of the sinuses of Valsalva. $^{3}$ Although we know that free ITA grafts yield excellent patency rates of approximately $90 \%$ at 7 years, the major problem influencing early free ITA graft patency was related to construction of a proximal anastomosis on the ascending aorta. In the case of CCBG, a segment of the free LITA graft at the site of proximal anastomosis matches better with the lower rate of pressure rise of the LAD artery than the ascending aorta. We strongly believe that with restoration of physiologic coronary flow in the diseased LAD region, we can expect an even better patency rate of CCCs incorporated into the LAD coronary bed.

\section{References}

1. Nottin R, Grinda JM, Anidjar S, Folliguet T, Detroux M. Coronarycoronary bypass graft: an arterial conduit-sparing procedure. J Thorac Cardiovasc Surg. 1996;12:1223-30. 
2. Nežić D, Knežević A, Borović S, Ćirković M, Milojević P. Coronarycoronary free internal thoracic artery graft on a single, distal, left anterior descending artery lesion. J Thorac Cardiovasc Surg. 2004;127:1517-8.

3. Biglioli P, Almanni F, Antona S, Sala A, Susini G. Coronary-coronary bypass: theoretical basis and techniques. J Cardiovasc Surg. 1987;28: 333-5.
4. Mariscalco G, Blanzola C, Leva C, Bruno V, Luvini M, Sala A. 19-year patency of a coronary-coronary venous bypass graft. Tex Heart Inst J. 2005;32:583-5.

5. Barboso G, Rusticali F. Proximal internal mammary in situ graft and distal coronary-coronary graft to revascularize left anterior descending coronary artery. Tex Heart Inst J. 2000;27:70-1.

\title{
Subvalvular aortic stenosis: Comprehensive cardiac evaluation with dual-source computed tomography
}

\author{
Paul Stolzmann, MD, ${ }^{a}$ Hans Scheffel, MD, ${ }^{a}$ Dominique Bettex, MD, ${ }^{b}$ Christoph Karlo, ${ }^{a}$ Thomas Frauenfelder, MD, \\ René Prêtre, MD, ${ }^{\mathrm{c}}$ Borut Marincek, MD, and Hatem Alkadhi, MD, ${ }^{a}$ Zurich, Switzerland
}

丹 Supplemental material is available online.

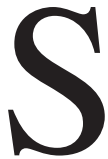

ubvalvular aortic stenosis (SAS) represents a fixed obstruction of the left ventricular outflow tract (LVOT) by an endocardial fold, fibrous tissue, or fibromuscular ridge. Echocardiography including color Doppler is the modality of choice to establish the diagnosis and to assess the extent and hemodynamic severity of SAS. ${ }^{1}$ We present imaging findings of a cardiac dual-source computed tomographic (CT) examination in a patient with SAS to demonstrate the potential of the noninvasive modality for a comprehensive evaluation of patients with the disease.

\section{Clinical Summary}

A 40-year-old woman was referred for follow-up after resection of a SAS 32 years ago. Clinical examination revealed a systolic grade 3/6 heart murmur and electrocardiography (ECG) showed stressinduced ST-segment depressions. Echocardiography demonstrated recurrence of the SAS causing LVOT obstruction (mean systolic pressure gradient $50 \mathrm{~mm} \mathrm{Hg}$ ). In addition, mild aortic regurgitation and prolapse of the anterior mitral valve leaflet were diagnosed.

From the Institute of Diagnostic Radiology and Institute of Anesthesia, Division of Cardiovascular Anesthesia, ${ }^{\mathrm{b}}$ and the Clinic for Cardiovascular Surgery, ${ }^{\mathrm{c}}$ University Hospital, Zurich, Switzerland.

This research has been supported by the National Center of Competence in Research, Computer Aided and Image Guided Medical Interventions of the Swiss National Science Foundation.

Received for publication Feb 27, 2007; accepted for publication March 8, 2007

Address for reprints: Hatem Alkadhi, MD, Institute of Diagnostic Radiology, University Hospital Zurich, Raemistrasse 100, 8091 Zurich, Switzerland (E-mail: hatem.alkadhi@usz.ch).

J Thorac Cardiovasc Surg 2007;134:240-2

$0022-5223 / \$ 32.00$

Copyright $\odot 2007$ by The American Association for Thoracic Surgery doi:10.1016/j.jtcvs.2007.03.005
Thus, the patient was advised to undergo surgical resection of the SAS with aortic valve replacement.

Preoperative workup included a CT coronary angiographic examination for exclusion of coronary artery disease. The patient underwent retrospective ECG-gated dual-source CT (Definition, Siemens Medical Solutions, Erlangen, Germany) after intravenous administration of $65 \mathrm{~mL}$ of iodinated contrast medium (Ultravist 300; Schering, Berlin, Germany). CT accurately depicted the fibromuscular ridge with a muscular base at the crest of the interventricular septum causing significant narrowing of the LVOT (Figure 1). The tricuspid aortic valve showed homogeneous thickening of the cusps and a coaptation defect in mid-diastole indicating aortic regurgitation (Figure 2, A). In addition, billowing of the anterior mitral valve leaflet in early systole indicated mitral valve prolapse (Figure 2, B). Left ventricular myocardial mass was elevated (308 g, adjusted: $178 \mathrm{~g} / \mathrm{m}^{2}$ ), whereas end-systolic (21 $\mathrm{mL})$ and end-diastolic volume $(120 \mathrm{~mL})$ as well as ejection fraction $(83 \%)$ were within normal limits. The coronary artery tree could be depicted with diagnostic image quality and showed no significant coronary stenosis (Figure E1).

Subsequently, the patient was reoperated on by a modified Konno procedure. The SAS was resected and the aortic valve was replaced. The postoperative course was uneventful and the patient was discharged to a rehabilitation facility on the eleventh postoperative day.

\section{Discussion}

The peculiar anatomy of the LVOT formed by the anterior, septal, and posterior walls of the left ventricle and by the anterior leaflet of the mitral valve gives rise for various and complex variants of SAS. They may present as thin membrane consisting of an endocardial fold and/or fibrous tissue, as a fibromuscular ridge consisting of a thickened membrane with a muscular base at the crest of the interventricular septum, as a fibromuscular ring or collar that is circumferentially attached to the LVOT and to the base of the anterior mitral valve leaflet, or as a fibromuscular ridge causing tunnel-like narrowing of the LVOT.

Precise knowledge of SAS morphologic characteristics is a requisite for successful surgery, and the extent of SAS has been shown to be associated with postoperative remodeling of the LVOT complex. ${ }^{2}$ Our case study demonstrates that CT enables a 